2000 Beam Instrumentation Workshop

Cambridge, MA, May 8-11, 2000

\title{
SNS Project-Wide Beam Current Monitors ${ }^{1}$
}

\author{
M. Kesselman ${ }^{*}$, R. Witkover ${ }^{* *}$, L. Doolittle ${ }^{* * *}$, J. Power $^{* * *}$ \\ "BNL, Upton, NY 11973, "** TechSource, Inc., Santa Fe, NM 87594, "** LBNL \\ Berkeley, CA 94720, ${ }^{* * * *}$ LANL Los Alamos, NM 87545
}

\begin{abstract}
A consortium of national laboratories is constructing the Spallation Neutron Source ${ }^{1}$ (SNS) to be installed at Oak Ridge National Laboratory. There are signal similarities that exist in the beam diagnostic instrumentation that could permit common designs. This paper will focus on the beam current monitoring requirements, and the methods under consideration to measure beam current in various locations throughout the SNS facility.
\end{abstract}

\section{REQUIREMENTS}

The Front End, Linac and HEBT will have a 57mA peak current pulse of about 1 ms duration (macro-pulse) chopped at the Ring revolution frequency of $1.05 \mathrm{MHz}$ (mini-pulse). Mini-pulses consist of approximately a $645 \mathrm{nS}$ long bunch and a $300 \mathrm{nS}$ gap. The micro-pulse structure of $402.5 \mathrm{MHz}$, which exists in the Front End, Linac, and shortly after injection into the Ring, will not be observed with the Beam Current Monitors (BCMs). The Ring will accumulate this current over $1 \mathrm{mS}$, about 1000 minipulses, resulting in an average of about 57 amps at the end of the cycle. The RTBT will carry the current of the last turn in the Ring to the target. The electronics in the Ring and RTBT must operate over a signal range of 1000 to $1(60 \mathrm{~dB})$, however, the same basic circuitry used in the Front End, Linac and HEBT could be used with the addition of gain switching.

Transformer droop during the macro-pulse, which will cause a baseline shift, is the result of finite inductance and load resistance limiting the $L / R$ time constant. If more turns are added to increase inductance the sensitivity goes down, and resonances with winding capacitance and delay line effects will limit the high frequency response.

Additionally, the Front End and Linac have little available space, and the transformers must be mounted in close proximity to DC magnetic fields. Large, shielded transformers, needed to obtain high inductance and exclude stray fields, will not fit.

To observe the details of the mini-pulse, a fast response ( $1 \mathrm{nS}$ rise time with a droop rate of about $0.1 \% / \mathrm{uS}$ ) is required. For the macro-pulse current, a rise time of about $50 \mathrm{nS}$ and a droop of $0.1 \% / \mathrm{mS}$ is required. The distribution of transformers is shown in Table 1.

${ }^{1}$ Work performed under the auspices of the U.S. Department of Energy. 


Table 1. Beam Current Monitor Distribution ( ${ }^{*}$ not finalized)
\begin{tabular}{|c|c|c|}
\hline Location & Beam Pipe Diameter & Number of BCMs \\
\hline Front End & $7 \mathrm{~cm}$ Flange OD & 2 \\
\hline Linac & $2.5 \mathrm{~cm}, 3.0 \mathrm{~cm}, 8 \mathrm{~cm}{ }^{*} \mathrm{ID}$ & 13 \\
\hline HEBT & $12 \mathrm{~cm} \mathrm{OD}$ & 4 \\
\hline Ring & $21 \mathrm{~cm} \mathrm{OD}$ & 1 \\
\hline RTBT & $21 \mathrm{~cm} \mathrm{OD}$ & 5 \\
\hline
\end{tabular}

\section{SURVEY OF CURRENT MONITORING TECHNIQUES}

Passive:

This is a basic beam current transformer consisting of a high-permeability tape wound core, with a single winding which acts as the secondary and the beam as the primary. As described above, conflicting requirements for rise time and droop make it difficult to design a transformer suitable to meet both and have adequate sensitivity for the first turn. A transformer suitable for observing the macro pulse in the Ring was developed ${ }^{2}$, however the large size and low sensitivity preclude its use in the Front End and Linac.

Fast Current Transformer (FCT):

A passive transformer but optimized for high frequency response, as a result there is considerable droop during the macro-pulse. However, commercial versions are available which are well suited to observing the mini-pulse. For example, Bergoz ${ }^{3}$ offers a unit with sub-nanosecond rise time and $0.1 \% / \mu \mathrm{S}$ droop. When observing the macro-pulse the baseline will gradually shift such that the area above zero is equal to the area below zero (average $=$ zero). For the $645 \mathrm{nS}$ pulse with $300 \mathrm{nS}$ gap the steady state transformer response will have a baseline shift nearly $2 / 3$ the pulse. This baseline can be restored on a mini-pulse by mini-pulse basis using a fast baseline restoration technique, as discussed below, to make macro-pulse measurements.

Hereward (magnetic feedback):

This approach uses a transformer with active feedback to compensate for droop by sensing the change in magnetic field in the core and restoring it through an additional winding. This technique works well, but trades sensitivity for low frequency response. Estimates indicate that it will not be appropriate for this application.

Active-Passive ${ }^{4}$ :

This is an interesting approach, employing an amplifier to compensate for droop without affecting the basic transformer high frequency response, and requiring no 
additional windings. However, using the transformer in the feedback loop gives rise to stability problems as a result of phase shift in the feedback signal. This may show up when the amplifier is located a distance from the transformer as it is in SNS (with the exception of the Front End). Bergoz offers an ACCT based on this approach with a stated bandwidth of $6 \mathrm{~Hz}$ to $300 \mathrm{kHz}$ with cable lengths hundreds of meters long. This bandwidth will not meet the SNS requirements.

\section{Direct-Current Current Transformer (DCCT):}

This approach involves a magnetic circuit employing multiple windings, and a modulation technique to force the magnetic core into the non-linear region, generating harmonics of the modulation frequency. The second harmonic, which is proportional to the magnetic field passing through the aperture, is detected and amplified. Response down to DC can be obtained. By combining this with other high frequency transformers with suitable matching crossover filters, a wide band system can be obtained. Several manufacturers offer such a device, however, the high frequency response is not of sufficient bandwidth and DC response is not required for SNS. Average current will be obtained numerically from the pulse response.

Integrating Current Transformer (ICT):

Very fast (sub-nanosecond) current pulses can not be easily detected with a current transformer due to frequency dependent magnetic losses. The ICT was designed to avoid this by pre-integrating the signal on an internal capacitor, then transferring it to the core. This also lessens the burden on the electronics, which now need only respond to a 10 to $20 \mathrm{nS}$ rise time. Bergoz supplies such a unit and associated electronics, which also provides baseline restoration and an analog output proportional to the charge. To observe mini-pulses we require nanosecond rise time response. The sub-nanosecond response of the ICT is not necessary, and the $20 \mathrm{nS}$ response is inadequate.

\section{DISCUSSION}

The wide band information associated with the mini-pulse and the information associated with the macro pulse can be extracted from a single transformer with suitable rise time and droop characteristics. To analyze the detailed mini-pulse a fast transformer with droop of $0.1 \% / \mu \mathrm{S}$ can be utilized. The macro-pulse data can be observed using the same transformer if appropriate baseline restoration is provided to restore the drooping baseline on a turn by turn basis. A general block diagram is shown in Figure 1.

The input conditioning employs gain adjustment to handle the large dynamic range of the Ring and RTBT, and need not be employed for the Front End, Linac and HEBT. The baseline restoration is required in all cases, and feeds the conditioned signal to various output processors. High-speed data acquisition with appropriate circular buffers for data storage process the mini-pulse. Integration of the current for charge analysis, with an ability to hold the turn-by-turn result and perform a digital 
conversion is a common requirement for all lines. Provision for local viewing and a filtered output to a track and hold amplifier followed by digital conversion completes the package.

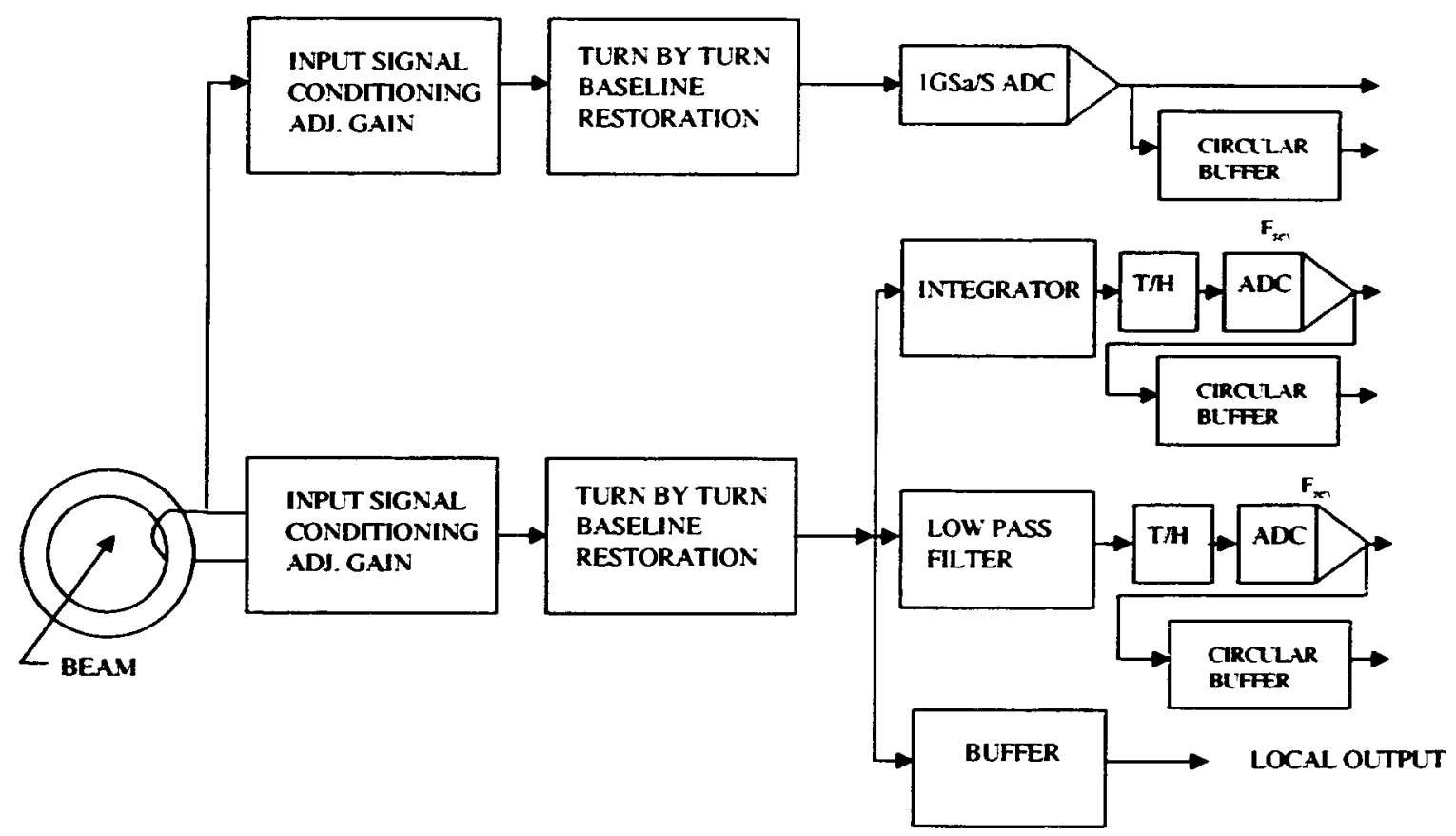

FIGURE 1. General block diagram

Input Signal Conditioning:

Two problems which must be resolved are: compensating for the signal droop due to finite $L / R$, and, noise reduction due to the remote location of the amplifier from the transformer. A single ended input can easily match a coaxial transmission line, but may suffer from noise induced on long cables. If a wide-band high common mode cable is used, a differential input stage can provide good common mode noise rejection and properly terminate the cable. A number of input conditioning amplifiers have been considered.

One approach suggested was to go directly into the negative input terminal of an opamp. Since the transformer serves as a current source, only the feedback resistor determines the op-amp output. In this case the droop time constant is given by the inductance $L$ divided by $R_{c}+R_{a}$, where $R_{c}$ is the coil resistance and $R_{a}$ is the feedback resistance divided by the open loop gain. This requires the op-amp be mounted directly at the transformer to avoid the need for cable matching resistors. Also, voltage noise is amplified by the full open loop voltage gain of the op-amp. At the high energy end of SNS, the radiation environment makes this option undesirable.

Gain switching will be necessary to accommodate the large signal range of the Ring and RTBT. A minimum of two gains is necessary for the RTBT: single turn 
capability, and full output capability. The Ring will require two or three gains, to cover the 1000:1 signal range. This would allow the signal to swing over a 10:1 range before switching gains and would maintain a digitizing error between $0.1 \%$ and $1 \%$. Additional gain settings could improve this. Fast electronic switches are available which would permit gain changes during the gap time, however, the transient caused by the gain change and any signal filtering could increase the settling time thereby corrupting data during the next turn. An alternate solution under consideration would employ the use of dual variable gain amplifiers, with switching after the amplifiers to allow observing the output of one while the other is changing gain.

Turn-by-Tum Base Line Restorer (BLR):

The finite L/R of the transformer will cause the baseline to gradually move away from zero during the mini-pulse (see Figure 2a for HEBT simulation and Figure $2 b$ for Ring simulation). A circuit is required which will acquire the signal during the gap time and subtract it from the signal during the beam time. This can be done either with an analog or digital circuit such as shown in Figures $3 a, 3 b$, and $3 c$. The BLR must respond quickly to permit sufficient settling of the signal electronics prior to the switched BLR operation. Approximately 7 time constants are required to settle to $0.1 \%$. For a $50 \mathrm{nS}$ rise time response $(\mathrm{Tau}=50 \mathrm{nS} / 2.2)$, about $160 \mathrm{nS}$ is required to achieve $0.1 \%$ settling. This provides about $140 \mathrm{nS}$ for the BLR to be switched on and settle which appears practical.

Analog BLR (see Figure 3a):

In the analog implementation the signal baseline is sampled just prior to the arrival of the first mini-pulse and then prior to successive mini-pulses, after the falling edge has settled. It is integrated for a finite time and held. By proper adjustment of the gain, via the inverting amplifier and the choice of gain in the summing stage, a voltage exactly opposite in amplitude to the output offset is added to the input signal. This restores the baseline to zero, and establishes the appropriate reference for the incoming signal pulse. This process is repeated for each successive mini-pulse, constantly readjusting the baseline for the total macro-pulse time. This readjustment helps to compensate for integrator drift during the macro-pulse. When the macro-pulse is completed the integrator is reset, and the process repeats.

Analog/Digital Hybrid BLR (see Figure 3b):

As in the analog BLR, the baseline is sampled just prior to the arrival of the minipulse. An ADC is used together with a DAC to provide a good representation of the baseline with no droop or drift as with the analog circuit described above. The digitized DC offset of the baseline is added to a digital accumulator to store and "remember" the offset level. By so doing, subsequent adjustments to the baseline offset are minimized, requiring only small (few bit) corrections. These corrections are added or subtracted to the previous offset stored in the accumulator, depending upon the sign of the ADC output. The DAC output is added to the input, however, due to the phase reversal a subtraction is actually performed. This process continues for the entire macro-pulse time, and is repeated at the start of the next macro-pulse. Unlike 
the analog version above, the digital accumulator need not be reset at the end of the macro-pulse, since there is no drift associated with the digital circuitry.

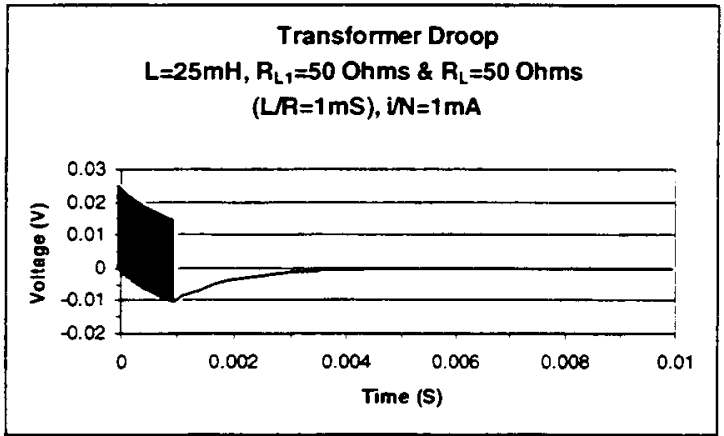

Figure 2a

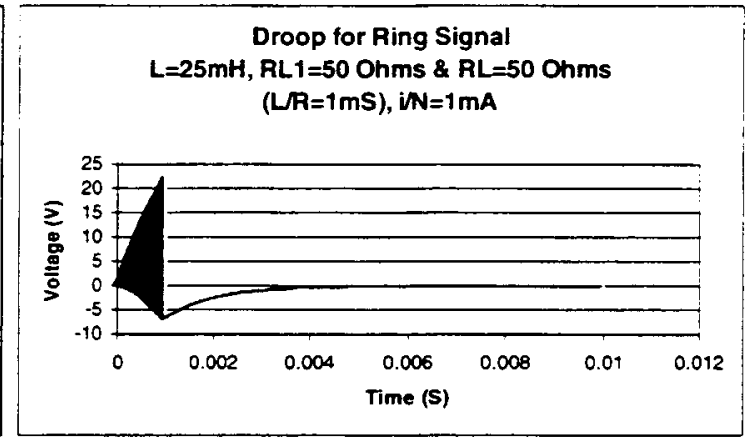

Figure 2b

Figure 2a. HEBT Current transformer response showing droop due to $L / R=1 \mathrm{mS}$. Pulse duration is $550 \mathrm{nS}$ and gap is $300 \mathrm{nS}$, repeated 1176 times. The transformer recovers to within $0.1 \%$ of the baseline in 7 time constants. $R_{L} 1$ is back termination. Figure $2 \mathbf{b}$. Ring Current transformer response showing droop due to $\mathrm{L} / \mathrm{R}=1 \mathrm{mS}$. Pulse duration is $550 \mathrm{nS}$ and gap is $300 \mathrm{nS}$, repeated 1176 times. The transformer recovers to within $0.1 \%$ of the baseline in 7 time constants. $\mathrm{R}_{\mathrm{L}} 1$ is back termination.

\section{Digital BLR (see Figure 3c):}

A fully digital implementation averages the signal during the gap time in the accumulator/divider. The average base line is stored and held in the storage register at the end of the gap time, and this is subtracted from the signal to restore the baseline to zero. The signal must be delayed in a circular memory to allow time for digital processing and proper alignment with the averaged base line calculation. The accumulator is reset prior to the calculation of the next turn's baseline. This technique suffers no drift, and could include additional digital processing techniques, such as insitu calibrated pole-zero cancellation and other filtering. In addition, it can be implemented in real-time on an FPGA, or as a post-processing step on a DSP or general-purpose computer.

\section{Integrator:}

The integrator used to measure the turn-by-turn (mini-pulse) charge must reset to zero (within a LSB) during the gap time of about $300 \mathrm{nS}$. This is insufficient for proper settling of the integrator, and a "ping-pong" approach is under consideration. This technique employs two integrators, resetting one while the other is integrating, effectively increasing the available reset time to $945 \mathrm{~ns}$. Circuitry to compensate for charge injection could be included but may be treated as an additional offset. The output of the integrator would be acquired during the gap-time by a fast track and hold and held until digitized by an ADC. The ADC data will be delivered to the control system and stored in a circular buffer suitable for holding 10 seconds of data. This buffer can be interrogated for later analysis.

An alternative design would use a fast 12 - 14 bit $\mathrm{ADC}$ operating at $30 \mathrm{MSa} / \mathrm{s}$ to $120 \mathrm{MSa} / \mathrm{s}$, sampling the current waveform and accumulating the result during the 
mini-pulse. This approach would depend on the availability of fast, high resolution $\mathrm{ADCs}$ at reasonable cost, but might be preferable to the analog integrator.

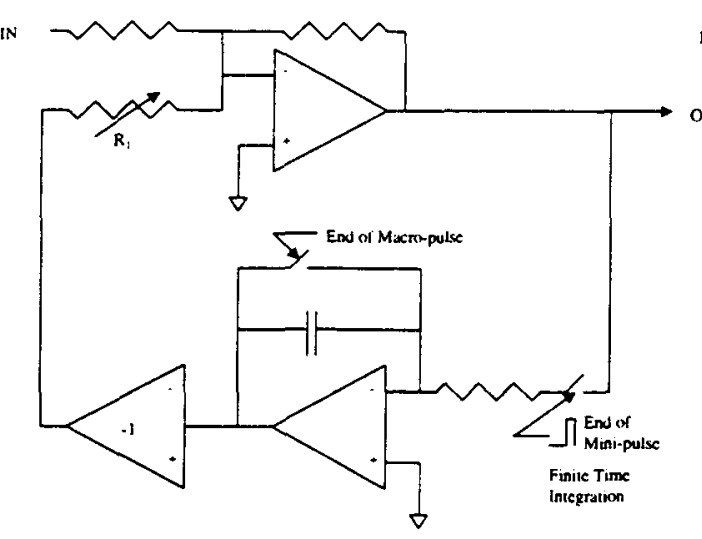

Figure 3a

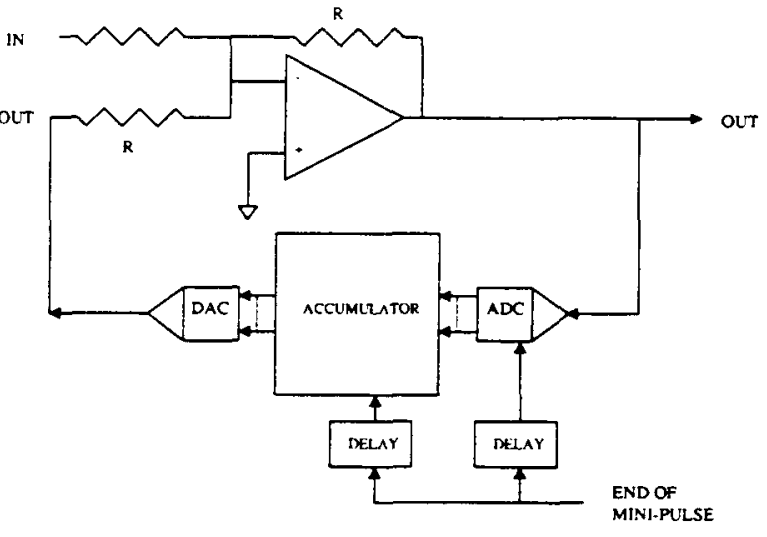

Figure 3b

Figure 3a. Analog turn by turn baseline restorer using finite time integration. A general BLR concept that may be implemented in different ways. Figure 3b. Hybrid Analog/Digital turn by turn baseline restorer. A general BLR concept that may be implemented in different ways.

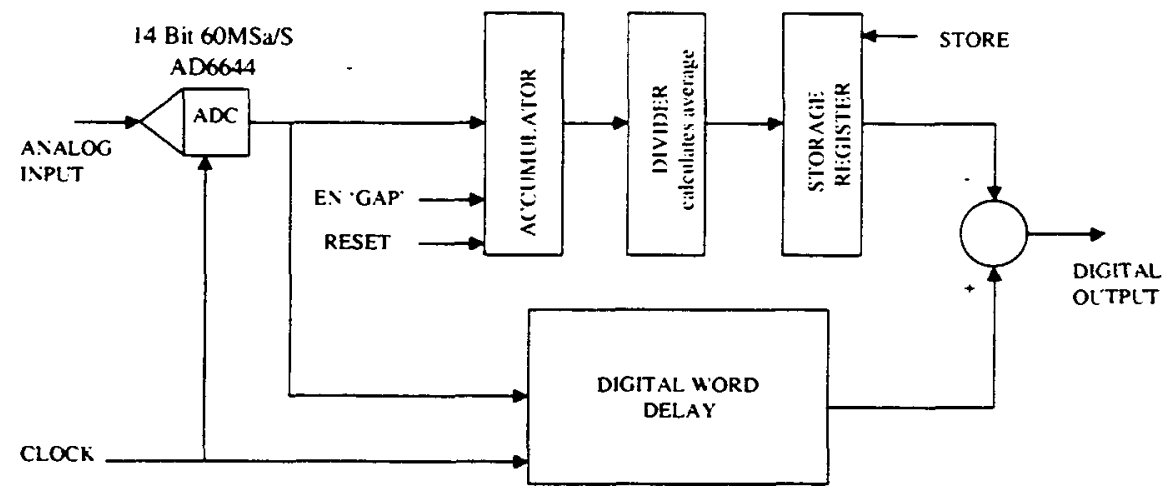

-Accumulator enabled only during 'GAP' time

- Baseline average stored in register \& Reset each turn

-ADC data delayed to allow time for baseline average calculation

Figure 3c. Fully Digital Implementation of BLR

Mini and Macro-pulse monitoring, and data storage:

In most every case where a current transformer is used, both a mini-pulse analysis, and a macro-pulse analysis is required. The RTBT line is an exception, requiring only mini-pulse analysis, and the MEBT line requiring only macro-pulse analysis. To process the mini-pulse, a $1 G S a / S$ ADC is shown in the block diagram of Figure 1. This converter will provide the basic data for digital integration to calculate charge, and will be stored in a circular buffer sufficient to hold 10 seconds of past historical data for analysis of failures. To accomplish this requires about $600 \mathrm{Mbytes}$ per transformer (ten to fifteen transformers are under consideration). The transmission of 
this data will place a severe burden on the network, even if only exercised off-line, and further discussion of operational requirements is needed.

Macro-pulse monitoring, depicted in figure 1, is filtered and acquired at the end of mini-pulses by a fast track and hold for conversion. The ADC data will be delivered to the control system and stored in a circular buffer suitable for holding 10 seconds of data. This buffer can be interrogated for later analysis.

An alternative design under consideration would share the same fast $12-14$ bit ADC discussed above. Additional digital processing could derive macro-pulse properties from that data. Again, values will be delivered to the control system, and will be stored in a circular buffer suitable for 10 seconds of data. This buffer can be interrogated for later analysis.

Local readout can be accomplished by a simple buffer amplifier, which will make the basic current signal available for viewing locally on an oscilloscope for diagnostic purposes.

\section{SUMMARY}

A number of approaches have been presented and require additional investigation. In particular, digital approaches seem to eliminate many analog difficulties, permitting drift free integration, filtering, and base line restoration. This seems most encouraging, particularly with ADCs becoming available which will provide 14 bit resolution at $65 \mathrm{MSa} / \mathrm{s}(\mathrm{AD} 6644)$.

\section{ACKNOWLEDGMENTS}

The author wishes to acknowledge the efforts of V. LoDesto and D. Kipp of BNL for their assistance with the construction and characterization of a test facility apparatus.

\section{REFERENCES}

1. http://www.sns.gov

2. Kesselman, M., Study of Ring BCM Requirements, Parameters and Feasibility, BNL/SNS Technote 062 , May 20,1999.

3. http//www.bergoz.com

4. Unser, K. B., Atomkemenergie, Kerntechnik, Vol. 47 (1985) No.1 (pg 48) 